\title{
Numerical Investigation of Plasma Sheath Under Multiple Oscillations in Acceleration Region of Hall Thrusters
}

\author{
Huijun Cao \\ Department of Mechanical and Automation Engineering, Xiamen City University, Xiamen, China \\ Email address: \\ huijun@xmcu.cn \\ To cite this article: \\ Huijun Cao. Numerical Investigation of Plasma Sheath Under Multiple Oscillations in Acceleration Region of Hall Thrusters. International \\ Journal of High Energy Physics. Vol. 4, No. 6, 2017, pp. 93-98. doi: 10.11648/j.ijhep.20170406.14
}

Received: October 20, 2017; Accepted: November 2, 2017; Published: December 25, 2017

\begin{abstract}
Oscillations range from $\mathrm{KHz}-\mathrm{GHz}$ have been measured in the experimental measurements within the laboratory Hall thruster. With the various frequency oscillations, different kinds of collisions between particles and the complex electromagnetic field environment, the dynamics of particles in the discharge chamber are really intricate. The dynamics of particles become even untraceable in the near-wall region where the plasma sheath exists. In this study, the two-dimensional fully kinetic Immersed Finite Element Particle-In-Cell (IFE-PIC) numerical models are developed in the axial-radial $(z-r)$ plane at the acceleration region, with the intent of examining the effects of multiple oscillations on the plasma sheath. The results are valuable for understanding the features of plasma sheath in acceleration region at the real working conditions.
\end{abstract}

Keywords: Plasma, Sheath, Multiple Oscillations, Full Particle-in-Cell

\section{Introduction}

The Hall thruster is one of the main electric propulsion equipment used in space. Since the first operative Hall thruster was launched to space in the 1970s, the Hall thruster has been tested on many satellites, and its performance has proved it as a reliable propulsion alternative [1-3]. The plasma sheath, which is an electric field arises at the boundary separating the plasma from a solid body in order to balance ion and electron currents at the collector surface, is an inherent phenomenon in Hall thruster [4-6]. Since the plasma sheath potential is related to the whole electric distribution in the discharge channel of Hall thruster and the sputter condition of the incident ions, the sheath is always a research focus among the physical problems of Hall thruster [5-6]. Moreover, as the most potential drop, the acceleration and sputtering of ions locate in the acceleration region, it is crucial to increase the understanding of the physical characteristics of plasma sheath in this region.

In the other hand, there have been many theoretical and experimental studies of oscillations in Hall thrusters. In general, oscillations can exist over a wide range of frequencies depending on values of parameters such as the discharge voltage and current, the magnetic field strength, the propellant flow, the number and location of the cathodes, the operating time since the thruster was last started, the total operating time of the thruster, the dynamic characteristics of the discharge power supply and the background pressure in which the thruster is operated [7-9]. The three typical oscillations in acceleration region are: the low frequency oscillation in whole channel; the ultra high frequency oscillation in exit region where the gradient of magnetic field is negative; the high frequency oscillation in near wall region [9], as shown in Figure 1. In this paper, the plasma sheath in acceleration region will be studied with consideration of the effects of these oscillations.

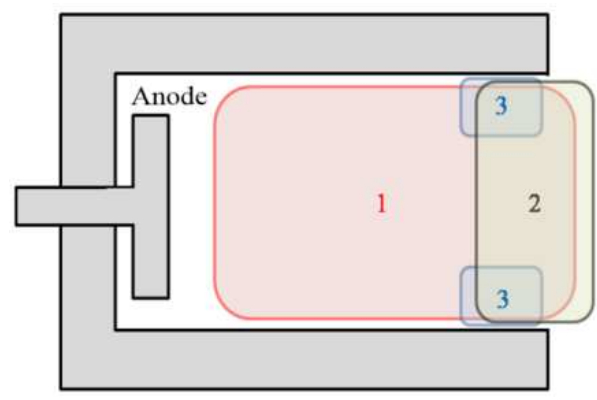

Figure 1. Schematic diagram of the zoning of oscillations in a Hall thruster: 1-the low frequency oscillation in whole channel; 2-the ultra high frequency oscillation in exit region where the gradient of magnetic field is negative; 3-the high frequency oscillation in near wall region. 


\section{Numerical Model}

Currently the most precise simulation model for plasma dynamic is based on the Particle-In-Cell (PIC) method, which utilizes a huge number of simulation particles for the plasma simulation and hence provides more specific description for the particle movements [10]. Compared with a unstructured grid, a structured grid will be more suitable for locating the huge number of simulation particles in the grid for PIC method. The immersed finite element (IFE) method which provides an efficient approach with convenient implementation to solve interface problems on structured grids independent of the interface [11], recently has been incorporated into the PIC method to propose the IFE-PIC method. The IFE-PIC method with IFE method solves the electric field based a Cartesian grid and the PIC method handles the movements and charge gathering of all simulation particles in the Cartesian grid, is widely used to study the plasma problems for ion-optical thruster $[12,13]$. In this paper, a two-dimensional fully kinetic IFE-PIC model is established to obtain investigate the plasma sheath under multiple oscillations in acceleration region of Hall thrusters.

\subsection{Domain and Boundaries}

The simulation object is the near-wall plasma sheath in acceleration region, as shown in Figure 2. Neumann boundary condition is applied at the left and right boundaries. The down side is the dielectric wall, while the up side is the quasineutral inlet in which the number of electrons and ions always keep the same. Ions colliding with the dielectric wall boundary are collected, whereas the electrons follow the secondary electron emission (SEE) model [14]. Particles that hit the other three boundaries are absorbed and deleted.

\subsection{Presets of the Electromagnetic Field}

The magnetic field intensity only has the uniform radial component $B_{r}$. The axial electric field intensity $E_{\mathrm{z}}$ with low frequency oscillation is utilized in this model:

$$
E_{\mathrm{z}}=E_{\mathrm{za}}+A \sin \left(2 \pi f_{z} \cdot t\right)
$$

where $E_{z a}$ is the time-average electric field intensity, $A$ is the amplitude, $f_{z}$ is the frequency which value equals the frequency of the low frequency oscillation. For SPT- $100^{3}$, the radial magnetic field intensity $B_{r}=300 \mathrm{Gauss}$ and the time-average electric field intensity $E_{\mathrm{za}}=2 \times 10^{4} \mathrm{~V} / \mathrm{m}$.

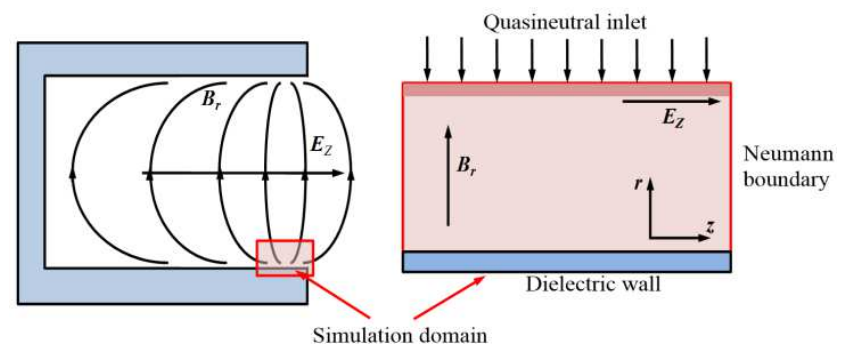

Figure 2. Sheath model at z-r plane.

\subsection{Field Solver}

The electric potential at each time-step is related to the charge density using Poisson's equation:

$$
\nabla^{2} \Phi=\frac{q_{e}}{\varepsilon_{0}}\left(n_{i}-n_{e}\right)
$$

where $\Phi$ denotes the electric potential, $q_{e}$ is the charge quantity, $\varepsilon_{0}$ denotes the vacuum permittivity, $n_{i}$ and $n_{e}$ are the densities of the ions and electrons. The electric field is determined from the electrical potential using $E=-\nabla \Phi$. At each step the electric field of the domain is solved and the number of ions and electrons in each cell is determined. The $n_{i}$ and $n_{e}$ are then obtained using the PIC weighting method and the charge density of each macro particle in the region of the four grid nodes [10].

The movements of the particles are determined from the integration of the equation of motion, which can be described using Newton's second law as [10]:

$$
\begin{gathered}
F=m \frac{d \mathrm{v}}{d t}=q(E+v \times B) \\
\frac{d x}{d t}=v
\end{gathered}
$$

where $F, m, q, v$ and $x$ are the force, mass, charge, velocity and position of the charged particle, $t$ is time, $E$ and $B$ are the local electric and magnetic field intensities, respectively.

\subsection{SEE Model}

The SEE and electric field models along dielectric walls, proposed by Morozov [14], are used in this research. The secondary electron yield $\chi$ is a function of the primary electron energy [15]:

$$
\chi(\zeta)=\chi(0)+\frac{\zeta}{\zeta^{*}}[1-\chi(0)]
$$

where $\zeta$ is the energy of a primary electron, $\chi(0)$ is the asymptotic total electron emission yield and $\zeta^{*}=53 \mathrm{eV}$ is the crossover energy for $\mathrm{BN}-\mathrm{SiO}_{2}$ ceramic [15].

According to Morozov [14], the electric field intensity at the dielectric surface can be expressed as:

$$
E_{\perp}=-\nabla \Phi=-\frac{\sigma}{\varepsilon_{0}}
$$

where $E_{\perp}$ presents the electric field intensity perpendicular to the wall, and $\sigma$ is the surface charge density collected by the wall.

\subsection{Scaling and Normalization}

Full kinetic PIC simulation is numerically infeasible because the high densities of the particles and the excessively 
short Debye length of the plasma in the Hall thruster [16]. Furthermore, simulating the ions and electrons simultaneously in one model is extremely difficult because the mass of the ion is about 240,000 times that of the electron. To simulate the ions and electrons simultaneously and make the computation feasible, the super-particle and artificial mass ratio are employed in the simulations. The above simplification procedures are commonly used to model physical problems in Hall thruster [17]. The manipulation and effects of the scaling model are detailed as follows.

Super-particles are applied in lieu of tracking real physical particles [10], where one super-particle represents about $2 \times 10^{7}$ real particles in the simulations. The mass of the ion is scaled by an artificial mass ratio factor $f(f>1)$ which means the ions are $f$ times lighter than that they should be. The artificial mass ratio factor $f=100$ is employed in this simulations, which make the ions move $\sqrt{f}=10$ times faster than in reality. This means the convergence time of the simulation could decrease by $\sqrt{f}=10$ times [16].

Table 1. Parameters utilized in the simulations.

\begin{tabular}{ll}
\hline Parameters & Value \\
\hline Plasma density & $10^{18} \mathrm{~m}^{-3}$ \\
Electron temperature & $10-20 \mathrm{eV}$ \\
Ion temperature & $1 \mathrm{eV}$ \\
Domain size & $100 \lambda_{d} \times 100 \lambda_{d}$ \\
\hline
\end{tabular}

The parameters used in the simulations are summarized in Table 1. All the variables in the model have been normalized to enable better interpretation of the simulation results. These variables and their normalized values are listed in Table 2 . The time-step $d t$ and grid spacing $d x$ are restricted by the electron gyro frequency $\omega_{c e}$ and the Debye length $\lambda_{d}$. Here, $d t=0.1$ $\omega_{c e}$ and $d x=0.5 \lambda_{d}$ are chosen in this simulation. The grid in the model consists of 200 cells in the axial direction and 200 cells in the radial direction.

Table 2. Reference variables.

\begin{tabular}{lll}
\hline Variable name & Reference/Unit & Value \\
\hline Mass & $m_{r e f} / \mathrm{kg}$ & $9.1 \times 10^{-31}$ \\
Charge & $q_{\text {ref }} / \mathrm{C}$ & $1.602 \times 10^{-19}$ \\
Length & $L_{r e f} / \mathrm{m}$ & $2.35 \times 10^{-5}$ \\
Time & $t_{r e f} / \mathrm{s}$ & $1.763 \times 10^{-11}$ \\
Velocity & $v_{r e f} / \mathrm{m} \mathrm{s}^{-1}$ & $1.326 \times 10^{6}$ \\
Potential & $\Phi_{r e f} / \mathrm{V}$ & 10 \\
Particle density & $n_{r e f} / \mathrm{m}^{-3}$ & $1.0 \times 10^{18}$ \\
Electric permittivity & $\varepsilon_{r e f} / \mathrm{F} \cdot \mathrm{m}^{-1}$ & $1.416 \times 10^{-8}$ \\
Temperature & $T_{r e f} / \mathrm{eV}$ & 10 \\
\hline
\end{tabular}

\section{Results and Discussion}

Firstly, we considered the ideal state that the axial electric field intensity is constant $(A=0)$. Figure 3 and Figure 4 show the distribution of sheath potential and ion density in sheath region in three different inject electron temperatures $(10 \mathrm{eV}$, $15 \mathrm{eV}$ and $20 \mathrm{eV}$ ), respectively. The sheath potential and the ion density in the sheath region are all showing as classic sheath form as shown in Figure 3 and Figure 4. So we can deduce that when the axial electric field intensity is constant, it had no effect on the physical properties of wall sheath. As the axial electric field intensity affects the axial velocity of particle, and sheath is made up of accumulated charge which is brought by particles incident on the wall. Potential drop of the sheath is in the radial direction. Constant velocity of the particle in the axial direction will not affect movement in the radial direction. Thus, the sheath performs the classic sheath form when the axial electric field intensity is constant.

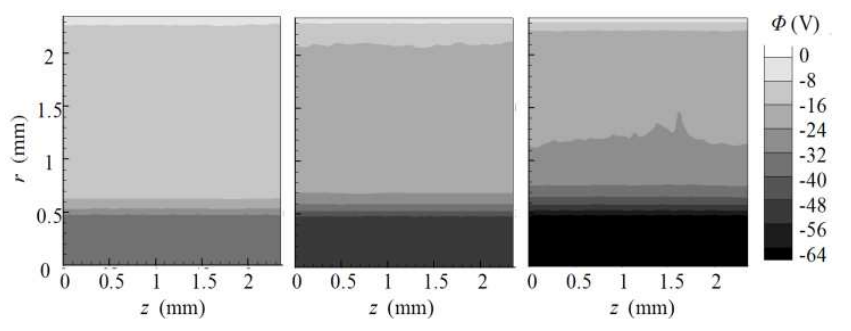

a) With $\mathrm{Te}=10 \mathrm{eV}$ b) With $\mathrm{Te}=15 \mathrm{eV}$ c) With $\mathrm{Te}=20 \mathrm{eV}$

Figure 3. Potentials with different inject electron temperatures.
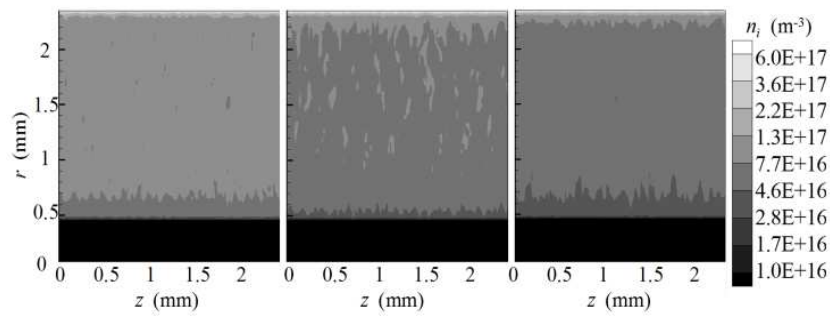

a) With $T e=10 \mathrm{eV}$ b) With $\mathrm{Te}=15 \mathrm{eV}$ c) With $\mathrm{Te}=20 \mathrm{eV}$

Figure 4. Ion densities in sheath region with different inject electron temperatures.

Next, we consider the actual situation which includes low frequency oscillation. The amplitude of Field harmonic oscillation $A$ is 0.1 times of the time average electric field intensity. The oscillation frequency $f_{z}$ is $f_{z}=10^{-6} \omega_{p e}$, whose magnitude order is $10^{4} \mathrm{~Hz}$. Thus, the equation 1 can be rewritten as:

$$
E_{\mathrm{z}}=E_{\mathrm{za}}+0.1 E_{\mathrm{za}} \cdot \sin \left(2 \pi \cdot 10^{-6} \cdot t\right)
$$

Figure 5 shows the distribution of sheath potential in three different inject electron temperatures $(10 \mathrm{eV}, 15 \mathrm{eV}$ and $20 \mathrm{eV})$ considered the axial oscillation electric field stated above, respectively. As the figure shows, when the inject electron temperature is $10 \mathrm{eV}$, the sheath is showing as significant oscillations sheath. When the inject electron temperature is 15 $\mathrm{eV}$, the potential amplitude has significantly reduced. As the inject electron temperature increases to $20 \mathrm{eV}$, the sheath is basically stable and no significant oscillations sheath can be observed. The potential distribution and the total potential drop in the situation that the inject electron temperature is 20 $\mathrm{eV}$ are basically the same as the result shown in previous ideal state $(A=0)$. As the inject electron temperature increases, the 
sheath oscillation is suppressed. When the electron temperature is high enough, the response speed of the electrons is sufficient to shield external interference. Then the sheath distribution alters to a steady state.
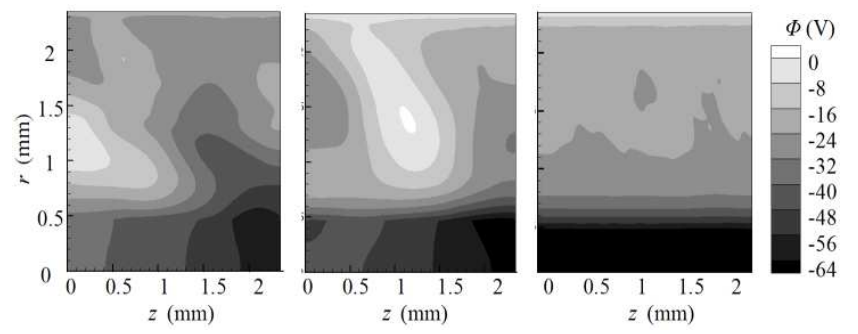

a) With $T e=10 \mathrm{eV}$ b) With $T e=15 \mathrm{eV}$ c) With $T e=20 \mathrm{eV}$

Figure 5. Potentials with different inject electron temperatures.

Figure 6 shows the distribution of ion density in sheath region in three different inject electron temperatures $(10 \mathrm{eV}$, $15 \mathrm{eV}$ and $20 \mathrm{eV}$ ) considered the axial oscillation electric field stated above, respectively. When the inject electron temperature is $10 \mathrm{eV}$, the ion density oscillation in the sheath is very severe. As the inject electron temperature increases to 20 $\mathrm{eV}$, the ion density is almost stable.
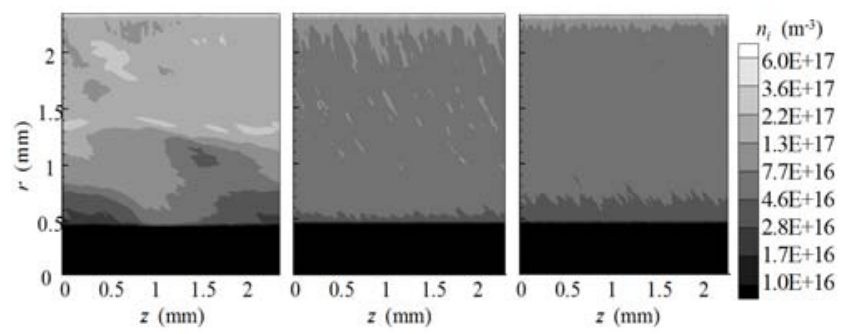

a) With $T e=10 \mathrm{eV}$ b) With $\mathrm{Te}=15 \mathrm{eV}$ c) With $\mathrm{Te}=20 \mathrm{eV}$

Figure 6. Ion densities in sheath region with different inject electron temperatures.

In order to clearly describe the potential change of oscillation sheath in the inject electron temperature of $15 \mathrm{eV}$, we record the potential profile of the point locates $0.0235 \mathrm{~mm}$ above the wall, as shown in Figure 7. From the figure we can obtain that the potential vigorous shakes over time and satisfies with simple harmonic. At the same time, we enlarge the potential oscillation curve which is shown in the red box on the right of Figure 7. It can be obtained that there is another high-frequency oscillation in the sheath at a finer time scale. Figure 8 shows ion density profile of the same point which is chosen in Figure 7. Form the figure we can see that the oscillation of ion density is more intense and the violent simple harmonic is barely to be observed. The difference between ion density amplitude and potential amplitude is probably because of that the ion density is the differential term of the potential.

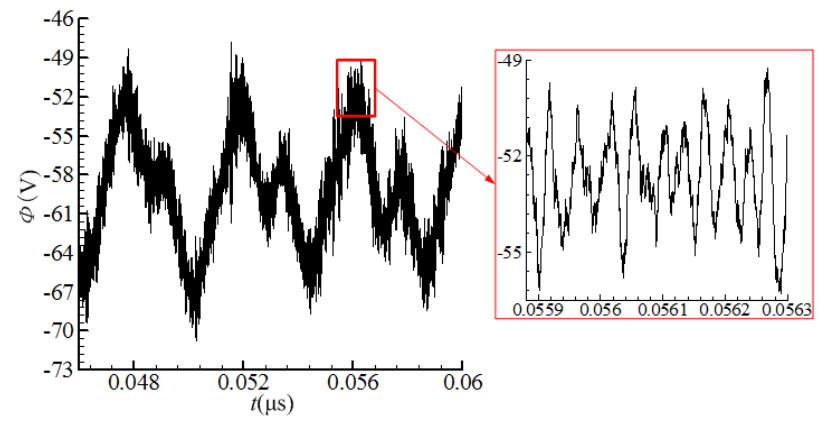

Figure 7. Potential profile of point which located at $z=0.235 \mathrm{~mm}$.

In order to get a more intuitive oscillation laws of the physical characteristics of sheath, we analysis the frequency of potential oscillations and ion density oscillations using the fast Fourier transform. The results show that there is no difference between the two kinds of spectrum. So, the Figure 9 gives out the oscillation spectrum of sheath potential which is shown in Figure 7. The potential oscillation frequency in high frequency region is $35 \mathrm{MHz}$, and in UHF band is $3.5 \mathrm{GHz}$. The Ultra-high frequency oscillation is the plasma oscillation caused by disturbance of electronic shell. While, the former high-frequency oscillation, whose frequency is different from the oscillation frequency of the applied electric field and far less than the plasma oscillation frequency, has larger amplitude and satisfy the laws of simple harmonic oscillation. Therefore, we think that when there is low-frequency oscillation electric field in axial direction and the electron temperature is low, the sheath potential not only retain the inherent high frequency oscillation but also stimulate structure oscillation that significantly different from the current oscillation frequency in the near wall region.

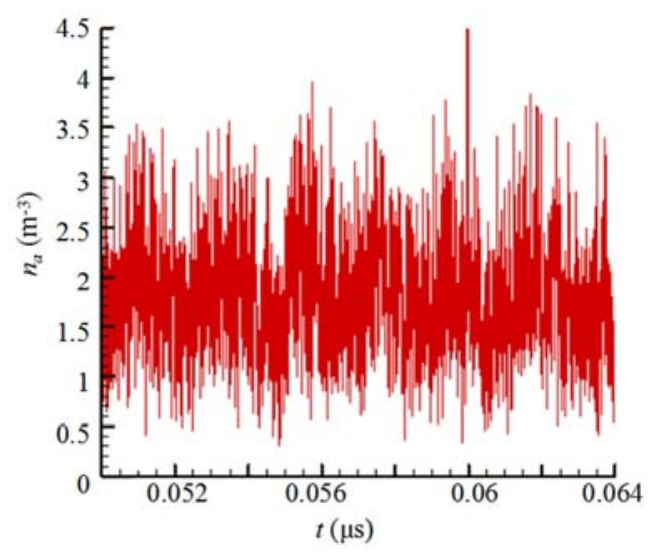

Figure 8. Ion density profile of point which located at $z=0.235 \mathrm{~mm}$. 


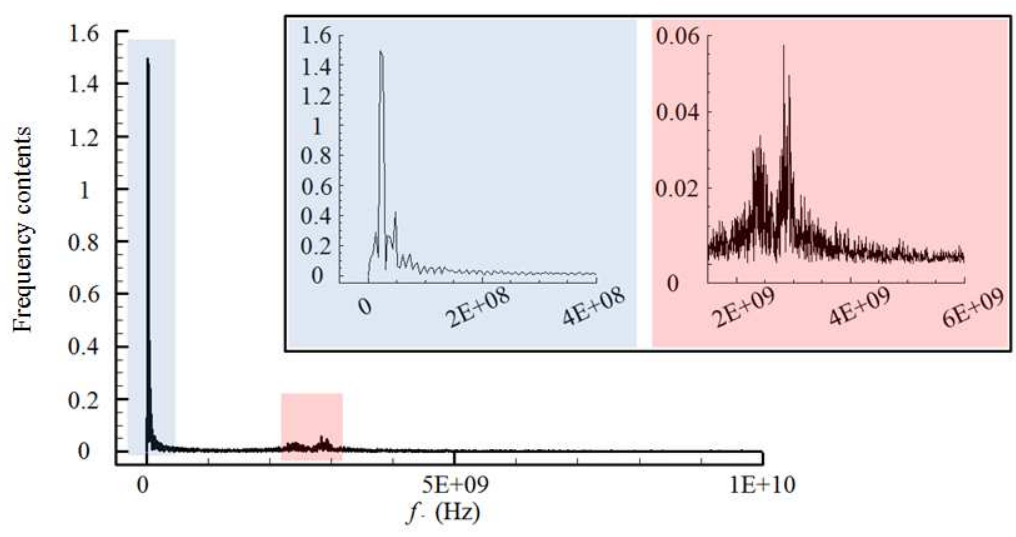

Figure 9. Frequency contents of sheath potential.

According to analysis the frequency range of Hall thruster observed in experiments, we can find out that this frequency value is consistent with the range of high frequency oscillation (1-100 MHz). The high-frequency oscillation located at channel outlet is caused by Kelvin-Helmholtz type shear flow of electronic transverse shear motion. In addition, the effect of external axial oscillating electric field on electrons also can be considered as transverse shear motion in the axial direction. Therefore, they perform the same oscillation frequency.

\section{Conclusion}

In this paper, the two-dimensional fully kinetic IFE-PIC numerical models are developed in the $z-r$ plane to investigate the effects of multiple oscillations on the plasma sheath. The following conclusions can be drawn:

1. The constant axial electric field have no effect on the physical properties of wall sheath; the velocity of the particles also have no change in the radial direction.

2. The sheath will show significant structure oscillations with a axial oscillation electric field and a lower electron temperature. This high-frequency oscillation may be caused by Kelvin-Helmholtz type shear flow of electronic transverse shear motion. When the electron temperature increases to a higher value, the plasma sheath with the high frequency oscillation will become to the classic sheath.

\section{Acknowledgements}

This work was financially supported by the University Distinguished Young Research Talent Training Program of Fujian Province under Grant No. JAT160835, and the Technology Project of Education Department of Fujian Province under Grant No. JAT160831.

\section{References}

[1] Hofer R. R., Jankovsky R. S., Gallimore A. D., High-Specific Impulse Hall Thrusters, Part 1: Influence of Current Density and Magnetic Field [J]. Journal of Propulsion and Power, 2006, 22 (4): 721-731.
[2] Hofer R. R., Jankovsky R. S., Gallimore A. D., High-Specific Impulse Hall Thrusters, Part 2: Efficiency Analysis. Journal of Propulsion and Power, 2006, 22 (4): 732-740.

[3] Goebel D. M., Katz I., Fundamentals of Electric Propulsion: Ion and Hall Thrusters. New Jersey: John Wiley \& Sons Inc. Publication, 2008: 325-443.

[4] Beilis I. I., Keidar M., Sheath and Presheath Structure in the Plasma-Wall Transition Layer in an Oblique Magnetic Field. Physics of Plasmas. 1998, 5 (5): 1545-1553.

[5] Morozov A. I., Savelyev V. V., One-Dimensional Model of the Debye Layer near a Dielectric Surface. Plasma Physics Reports. 2002, 28 (12): 1017-1023.

[6] Morozov A. I., Savelyev V. V., Structure of Steady-State Debye Layers in a Low-Density Plasma near a Dielectric Surface [J]. Plasma Physics Reports. 2004, 30 (4): 299-306.

[7] Esipchuk Y. B., Morozov A. I., Tilinin G. N., et al., Plasma Oscillations in Closed-Drift Accelerators with an Extended Acceleration Zone. Soviet Physics Technical Physics. 1974, 18: 928-1466.

[8] Baranov V., Nazarenko Y., Petrosov V., et al. Theory of Oscillations and Conductivity for Hall Thruster//32nd AIAA/SAE/ASME/ASEE Joint Propulsion Conference and Exhibit, AIAA-1996-3192, Lake Buena Vista, Florida, 1996.

[9] Choueiri E. Y., Plasma Oscillations in Hall Thrusters. Physics of Plasmas. 2001, 8 (4): 1411-1426.

[10] Birdsall C. K., Langdon A. B., Plasma Physics via Computer Simulation. Bristol: Adam Hilger, 1991: 207-479.

[11] Lin T., Lin Y., Sun W. W., et al. Immersed Finite Element Methods for 4th Order Differential Equations. Journal of Computational and Applied Mathematics, 2011, 235 (13): 3953-3964.

[12] Kafafy R. I., Immersed Finite Element Particle-In-Cell Simulations of Ion Propulsion. Virginia: Thesis for the Degree of Doctor of Philosophy. University of Virginia Polytechnic Institute and State. 2005: 29-55.

[13] Wang J., Cao Y., Kafafy R., et al. Numerical and Experimental Investigations of Crossover Ion Impingement for Subscale Ion Optics. Journal of Propulsion and Power, 2008, 24 (3): 562570.

[14] Morozov A. I., Steady-state uniform Debye sheaths [J]. Soviet Journal of Plasma Physics. 1991, 17: 393-396. 
[15] Barral S., Makowski K., Peradzynski Z., Gascon N., and Dudeck M., Wall material effects in stationary plasma thrusters. II. Near-wall and in-wall conductivity. Phys. Plasmas, 10 (10): 4137-4152, 2003.

[16] Szabo J., Fully kinetic numerical modeling of a plasma thruster, Massachusetts Institute of Technology, (2001).
[17] Liu H., Yu D. R., Yan G. J., and Liu J. Y., Investigation of the start transient in a Hall thruster. Contrib. Plasma Phys., 48 (9): 603-611, 2008. 